\title{
Visualization of Electrochemical Reactions by Redox-dependent Quenching of Photoluminescence from ZnS-AgInS 2 Solid Solution Semiconductor Nanoparticles
}

\section{Taro UEMATSU, ${ }^{a}$ Yusuke KAJI, ${ }^{a}$ Tsukasa TORIMOTO, ${ }^{b}$ and Susumu KUWABATAa,*}

a Department of Applied Chemistry, Graduate School of Engineering, Osaka University, Suita, Osaka 565-0871, Japan

b Department of Crystalline Materials Science, Graduate School of Engineering, Nagoya University, Chikusa-ku, Nagoya 464-8603, Japan

* Corresponding author: kuwabata@chem.eng.osaka-u.ac.jp

\begin{abstract}
A new method to visualize electrochemical reactions by fluorescence is demonstrated by using the photoluminescence quenching of semiconductor nanoparticles which changes according to the redox states of quenchers. The photoluminescence intensity of $\mathrm{ZnS}-\mathrm{AgInS}_{2}$ solid solution semiconductor nanoparticles can be controlled by the electrochemical manipulation of quencher molecules. By using a fluorescence optical microscopy, the formation of Nernst diffusion layer is successfully observed as a photoluminescence intensity gradient.

(C) The Electrochemical Society of Japan, All rights reserved.

Keywords : Semiconductor Nanoparticles, Photoluminescence Quenching, Nernst Diffusion Layer, Fluorescence Microscopy
\end{abstract}

\section{Introduction}

Semiconductor nanoparticles (NPs) in the size regime of Bohr radius show unique optical properties. ${ }^{1}$ A size-dependent bandgap variation and photoluminescence $(\mathrm{PL})$ at room temperature are typical phenomena that attract much research attention. ${ }^{2}$ Over the past 20 years, remarkable improvements on semiconductor NPs have been achieved; not only the synthetic methods but also post treatments to improve their functions have been proposed. ${ }^{3-6}$ Being supported by such numerous investigations, semiconductor NPs have been used as fluorophores in biochemistry. ${ }^{7}$

More recently, the detection of biological reactions by $\mathrm{PL}$ quenching caused by a fluorescence resonance energy transfer (FRET) have been reported, ${ }^{8-10}$ because the effective range of the FRET, typically $1-10 \mathrm{~nm}$, is adequate for the detection of proteinprotein interactions. ${ }^{11}$ On the other hand, PL quenching by a photoinduced electron transfer to redox species is known as another mechanism that has higher sensitivity as to detect the adsorption of a single molecule on one NP. ${ }^{12-17}$ The rate of electron transfer is very amenable to the changes in distance around $1 \mathrm{~nm}$ that is shorter than the distance needed for the FRET. We demonstrated that the polarity of both NPs and quenchers in aqueous solution profoundly affects the efficiency of the electron transfer by virtue of variations in the electrostatic force between the two species. ${ }^{12,13}$ It was also found that the reduction of a cationic quenchers resulted in the significant loss of its quenching ability, because the neutralization upon reduction eliminates the electrostatic interaction with anionic NPs. ${ }^{14}$ A glucose sensing was demonstrated by the charge variation of methyl phenazium cation upon its redox reaction in combination with an enzymatic reaction by glucose dehydrogenase.

The applicability of this type of detection method is not limited to the measurement of the targets' concentration, but it is also possible to know their spatial distribution. Herein, we demonstrate a new means to visualize electrochemical reactions by the fluorescence quenching of the ZnS-AgInS 2 solid solution (ZAIS) NPs caused by electron transfer as a probe. In situ techniques to observe an electrochemical diffusion layer had been reported using a specially modified microscopy with diode array detector, ${ }^{18-20}$ a confocal Raman microscopy, ${ }^{21-24}$ and recently by a scanning electron microscopy with an x-ray spectrometer. ${ }^{25}$ The present method detecting the variation in polarity can, in principle, cover many types of redox reactions if semiconductor NPs with an appropriate energy level and polarity are selected. In the present study, low toxic fluorescent semiconductor NPs, ZAIS NPs, are used. ${ }^{26,27}$ Preliminary experiments using a standard spectroelectrochemistry technique revealed that the control over the PL quenching by an electrochemical manipulation is successful. Then, a fluorescence microscopy was used to obtain the two-dimensional image of Nernst diffusion layer.

\section{Experimental}

\subsection{Materials and methods}

Silver nitrate, indium nitrate, zinc nitrate, sodium diethyldithiocarbamate, thioglycolic acid, $N, N, N^{\prime}, N^{\prime}$-tetramethylphenylenediamine (TMPDA), methylphenazium methansulfonate $\left(\mathrm{MP}^{+}\right)$, and palladium black were purchased from Wako Pure Chemical Industries and used without further purification. Oleylamine (Wako) was purified by distillation under reduced pressure $(100 \mathrm{~Pa})$ before use. 2-Aminoethanol and 3-mercaptopropionic acid were purchased from Tokyo Chemical Industry and purified by distillation. A platinum (99.99\%) wire, plate, and mesh used for electrodes were purchased from Nilaco Corporation. Water used in the series of experiments was purified by Milli-Q Integral 3 (Millipore, $18.2 \mathrm{MWcm}$ ). The synthesis of the ZAIS NPs and successive ligand exchange to 3-mercaptopropinoic acid (MPA) was conducted by a method reported recently. ${ }^{12}$ The resulting MPA-capped ZAIS (MPA-ZAIS) NPs were dissolved in water. $N, N, N^{\prime}, N^{\prime}$-tetramethylquinonediimine $\left(\mathrm{TMQDI}^{2+}\right.$ ) was produced by oxidizing TMPDA with oxygen in the presence of palladium black.

\subsection{Instrumental}

All the electrochemical control was performed with a potentiostat (HOKUTO DENKO, HA-151). Electrochemical PL intensity 
(a)

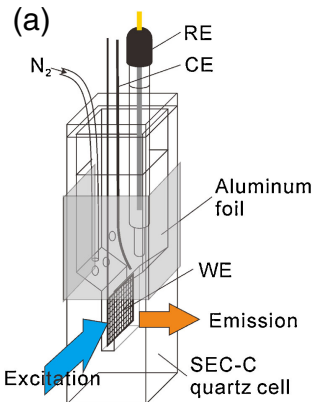

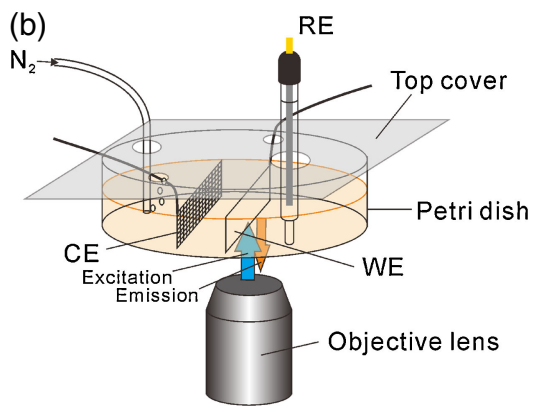

(b)

Figure 1. (Color online) Schematic illustration of spectroelectrochemistry setup for the detection of bulk PL intensity changes, (a). Electrochemical cell for the fluorescence microscope observation of Nernst diffusion layer upon the polarization of platinum working electrode, (b).

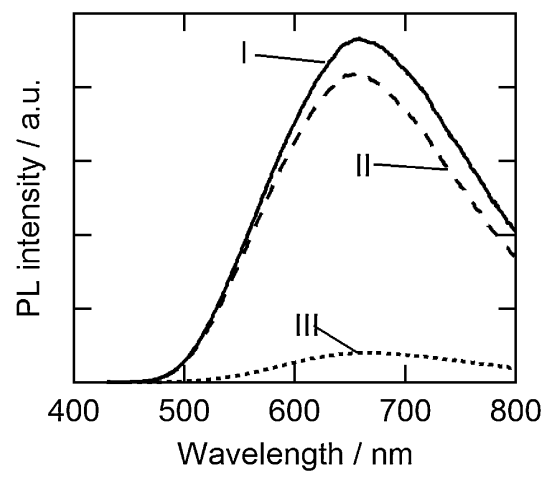

Figure 2. PL spectra of MPA-ZAIS NPs solution (I), MPA-ZAIS NPs in the presence of TMPDA $(30 \mu \mathrm{M}, \mathrm{II})$, and in the presence of $\mathrm{TMQDI}^{2+}(30 \mu \mathrm{M}, \mathrm{III})$. All samples were excited at $420 \mathrm{~nm}$.
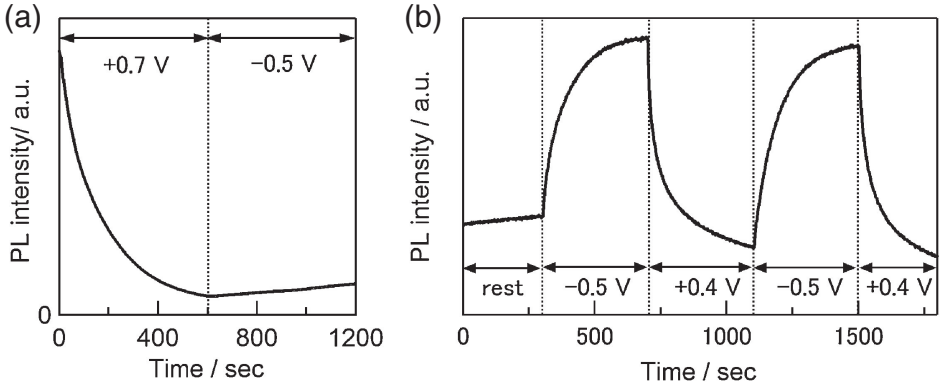

Figure 3. PL intensity changes of MPA-ZAIS NPs recorded with a setup illustrated in Fig. 1(a) during the successive reduction/oxidation of TMPDA $(30 \mu \mathrm{M}, \mathrm{a})$ and $\mathrm{MP}^{+}(10 \mu \mathrm{M}, \mathrm{b})$. Excitation and emission wavelengths were $420 \mathrm{~nm}$ and $635 \mathrm{~nm}$, respectively.

responses were measured in a commercial thin-layer quartz glass cell cuvette (BAS, SEC-C) filled with a phosphate buffered electrolyte $(\mathrm{pH}=7.0)$ containing the ZAIS NPs $(1 \mu \mathrm{M}$ in terms of particle) and quenchers $(10-30 \mu \mathrm{M})$. A platinum mesh inserted into a thin groove $(10 \mathrm{~mm} \times 10 \mathrm{~mm} \times 1 \mathrm{~mm})$ at the bottom of the cuvette served as a working electrode. A reference $(\mathrm{Ag} \mid \mathrm{AgCl})$ and counter electrodes were placed in the upper part of the cuvette, outside of which was covered with an aluminum foil to prevent the excitation of an unnecessary region outside the groove [Fig. 1(a)]. The cuvette was placed in a fluorospectrometer (Horiba Jobin Yvon, Fluoromax4) and filled with nitrogen. The time variation of fluorescence intensity was recorded during the polarization of the working electrode.

A fluorescence microscopy imaging was performed with a reflection inverted fluorescence microscopy (Nikon, ECLIPSE TE2000$\mathrm{U}$ ) equipped with a digital camera and a home-made electrochemical cell placed on a sample stage [Fig. 1(b)]. A platinum plate working electrode $(t=0.1 \mathrm{~mm})$ was aligned vertically at the bottom of the cell, and a platinum mesh counter electrode and $\mathrm{Ag} \mid \mathrm{AgCl}$ reference electrode were attached to the cell. The cell was covered with an acrylate resin plate and nitrogen was filled during experiments. Excitation light $(465-495 \mathrm{~nm})$ was irradiated through an objective lens, and fluorescence images were recorded by the digital camera.

\section{Results and Discussion}

Our recent studies have shown that the variation in the valence number of quenchers upon redox reaction and resulting changes in electrostatic affinity with NPs are main reasons for the difference in their quenching ability. Two possible quenchers were employed in the present study. TMPDA was selected with an expectation of significant increase in the quenching ability when it is oxidized to divalent $N, N, N^{\prime}, N^{\prime}$-tetramethylquinonediimine (TMQDI ${ }^{2+}$ ), as dem- onstrated in Fig. 2. The magnitudes of quenching evaluated as $I_{0} / I-1$ were 90 times different between its redox states, where $I_{0}$ and $I$ are PL intensity in the absence and presence of quenchers, respectively. Another candidate is monovalent $\mathrm{MP}^{+}$used in our previous investigation on glucose sensing, ${ }^{14}$ because $\mathrm{MP}^{+}$also loses the quenching ability by the reduction to neutral MPH.

Figure 3(a) shows the time course PL intensity variation of MPAZAIS NPs solution ( $1 \mu \mathrm{M}$ in terms of particle) in the presence of TMPDA $(30 \mu \mathrm{M})$ when the potential of a Pt mesh electrode illustrated in Fig. 1(a) was polarized at $+0.7 \mathrm{~V}$ vs. $\mathrm{Ag} \mid \mathrm{AgCl}$, which is sufficiently positive to oxidize TMPDA into TMQDI ${ }^{2+}$ having redox potentials of $+0.079 \mathrm{~V}$ for $\mathrm{TMPDA}^{+1 / 0}$ and $+0.552 \mathrm{~V}$ for TMPDA $^{2+/ 1+}$ (TMQDI). ${ }^{28}$ The NPs were quenched gradually with an increase in the TMQDI ${ }^{2+}$ concentration. However, even when the potential is switched to $-0.5 \mathrm{~V}$, the PL intensity did not recover. The irreversibility of quenching may derive from the strong adsorptive force of divalent quenchers to the NPs surface covered with carboxyl groups. Previous studies revealed that divalent quenchers have large adsorption coefficients as they can be detected when the equimolar of quenchers are added to the NPs solution. ${ }^{12,13}$ The adsorbed quenchers have very small chance to desorb from the NP even when the free quenchers surrounding the NP is reduced. Also, the direct reduction of the adsorbed quenchers is less likely because of 1-2 orders of magnitude lower diffusion coefficient of the NPs $\left(7 \times 10^{-11} \mathrm{~m}^{2} \mathrm{~s}^{-1}\right.$ calculated from Stokes-Einstein equation) than small ions (typically $1 \times 10^{-9} \mathrm{~m}^{2} \mathrm{~s}^{-1}$ ). Even if they approach to the electrode, a high steric hindrance prevent the electron exchange with the flat-plate electrode. On the other hand, when monovalent $\mathrm{MP}^{+}(10 \mu \mathrm{M})$ was used as a quencher, the reversibility of quenching could be attained, as evidenced from a reversible PL intensity switching by the polarization of the electrode indicated in Fig. 3(b). The initially quenched NPs recovered their PL upon the reduction of $\mathrm{MP}^{+}$to MPH when the platinum electrode [Fig. 1(a)] was polarized 
(a)

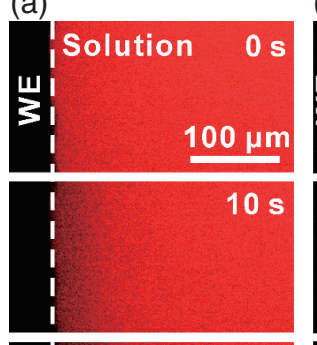

(b)

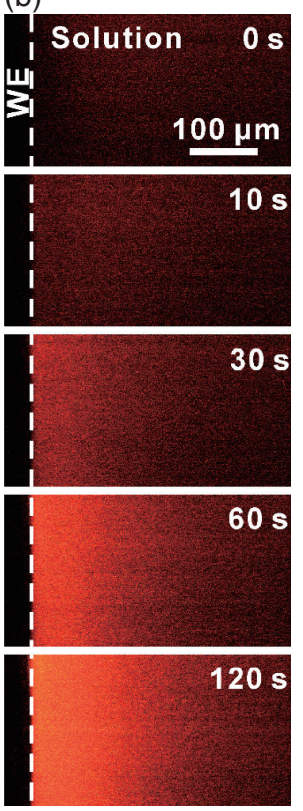

Figure 4. Fluorescence microscopy images recorded with a setup illustrated in Fig. 1(b) at the interface of Pt electrode and solution containing ZAIS NPs and quenchers: brightness variation when TMPDA is oxidized at $+0.7 \mathrm{~V}$ (a), and when $\mathrm{MP}^{+}$is reduced at $-0.5 \mathrm{~V}(\mathrm{~b})$

at $-0.5 \mathrm{~V}$. The reduction of free quenchers seemed to change the number of adsorbed quenchers due to the relatively weak adsorption coefficient of $\mathrm{MP}^{+}$compared with $\mathrm{TMQDI}^{2+}$. These results coincide with a previous finding that monovalent quenchers have moderate adsorption coefficients to the charged NPs and part of them remain free in a solution. ${ }^{13}$ The PL intensity decreased again when the potential is switched to $+0.4 \mathrm{~V}$, which cause the complete oxidation of MPH to $\mathrm{MP}^{+}$. The slight decrease in the PL intensity by a repeated potential switching might derive from the degradation of NPs themselves by the electrode.

Figure 4 shows fluorescence microscopy images obtained with the setup illustrated in Fig. 1(b). In Fig. 4(a), the platinum electrode was polarized at $+0.7 \mathrm{~V}$ in a phosphate buffered solution $(\mathrm{pH}=7)$ containing MPA-ZAIS NPs and TMPDA $(30 \mu \mathrm{M})$. The distinct shadows on the left side of the figures correspond to the edge of the platinum working electrode. The solution, uniformly glowing with orange at $0 \mathrm{~s}$, started to become dark from the vicinity of the electrode as $\mathrm{TMQDI}^{2+}$ is generated and spread out from the electrode surface. During $120 \mathrm{~s}$, the electrochemical diffusion layer was grown to more than $200 \mu \mathrm{m}$, which is comparable to a generally-accepted thickness of hundreds of micrometers. As expected from the result in Fig. 3(a), the recovery of PL did not occur for the TMPDA system by the reason mentioned in the last paragraph; a very slow emission recovery originated from the diffusion of ZAIS NPs themselves. The strong adsorption may also contribute to the diffusion layer thickness that is slightly thinner than the thickness observed for $\mathrm{MP}^{+}$, because a portion of electrochemically generated TMQDI ${ }^{2+}$ is trapped by the NPs of slow diffusion. The distinct recovery in the PL intensity by the electrochemical reaction was achieved by using monovalent $\mathrm{MP}^{+}(10 \mu \mathrm{M})$ as a quencher [Fig. 4(b)]. In this case, the PL recovered gradually from the electrode when it was polarized at $-0.5 \mathrm{~V}$, which is consistent with the result shown in Fig. 3(b). The reduction of free $\mathrm{MP}^{+}$lowered its concentration in the vicinity of the electrode over time, and the number of adsorbed $\mathrm{MP}^{+}$at each NP decreased according to the concentration ratio of $\mathrm{MP}^{+} / \mathrm{MPH}$ around the $\mathrm{NP}$, owing to the relatively low adsorption coefficient of monovalent quenchers. In such a manner, the spatial distribution of $\mathrm{MP}^{+}$was detected as the fluorescence intensity.

\section{Conclusion}

The electrochemical reactions were monitored in situ by using the redox-dependent PL quenching of semiconductor NPs as a probe. Judging from the irreversibility of the quenching observed for the divalent quencher, the interaction between the NP and the quencher should be moderate to the extent that the quencher can desorb from the NP in a short period. Although we attempted to estimate the absolute concentration of reaction species, the results did not have sufficient matching with digital simulations based on the conventional electrochemical theory. The following two points may contribute to the mismatch with the theory: the slow diffusion of redox species due to their adsorption onto NPs, and the scattering of light which blurs fluorescence microscope images. The first problem would be overcome by tuning the electrical charge of NPs using the mixture of ligands, and the second problem should be improved if a confocal microscopy is introduced. Further investigations to improve the accuracy of measurement is underway based on the aforementioned points.

\section{References}

1. A. M. Smith and S. Nie, Acc. Chem. Res., 43, 190 (2010).

2. D. V. Talapin, J. S. Lee, M. V. Kovalenko, and E. V. Shevchenko, Chem. Rev., 110, 389 (2010).

3. M. Geszke-Moritz and M. Moritz, Mater. Sci. Eng., C, 33, 1008 (2013).

4. M. Green, J. Mater. Chem., 20, 5797 (2010).

5. R. Xie, M. Rutherford, and X. Peng, J. Am. Chem. Soc., 131, 5691 (2009).

6. C. B. Murray, D. J. Norris, and M. G. Bawendi, J. Am. Chem. Soc., 115, 8706 (1993).

7. M. A. Walling, J. A. Novak, and J. R. E. Shepard, Int. J. Mol. Sci., 10, 441 (2009).

8. I. L. Medintz, A. R. Clapp, F. M. Brunel, T. Tiefenbrunn, H. T. Uyeda, E. L. Chang, J. R. Deschamps, P. E. Dawson, and H. Mattoussi, Nat. Mater, 5, 581 (2006).

9. F. A. Esteve-Turrillas and A. Abad-Fuentes, Biosens. Bioelectron., 41, 12 (2013).

10. R. Gill, M. Zayats, and I. Willner, Angew. Chem., Int. Ed., 47, 7602 (2008).

11. J. R. Lakowicz, Principles of Fluorescence Spectroscopy, Third Edition, Springer Science+Business Media, New York (2006)

12. T. Uematsu, A. Doko, T. Torimoto, K. Oohora, T. Hayashi, and S. Kuwabata, J. Phys. Chem. C, 117, 15667 (2013).

13. T. Uematsu, T. Waki, T. Torimoto, and S. Kuwabata, J. Phys. Chem. C, 113, 21621 (2009).

14. T. Uematsu, S. Taniguchi, T. Torimoto, and S. Kuwabata, Chem. Commun., 7485 (2009).

15. R. C. Mulrooney, N. Singh, N. Kaur, and J. F. Callan, Chem. Commun., 686 (2009).

16. I. L. Medintz, M. H. Stewart, S. A. Trammell, K. Susumu, J. B. Delehanty, B. C. Mei, J. S. Melinger, J. B. Blanco-Canosa, P. E. Dawson, and H. Mattoussi, Nat. Mater, 9, 676 (2010).

17. I. L. Medintz, T. Pons, S. A. Trammell, A. F. Grimes, D. S. English, J. B. BlancoCanosa, P. E. Dawson, and H. Mattoussi, J. Am. Chem. Soc., 130, 16745 (2008).

18. C. C. Jan and R. L. McCreery, J. Electroanal. Chem. Interfacial Electrochem., 220, 41 (1987).

19. C. C. Jan and R. L. McCreery, Anal. Chem., 58, 2771 (1986).

20. C. C. Jan, R. L. McCreery, and F. T. Gamble, Anal. Chem., 57, 1763 (1985)

21. C. Amatore, S. Szunerits, L. Thouin, and J. S. Warkocz, Electrochem. Commun., 2, 353 (2000).

22. C. Amatore, S. Szunerits, and L. Thouin, Electrochem. Commun., 2, 248 (2000).

23. C. Amatore, F. Bonhomme, J. L. Bruneel, L. Servant, and L. Thouin, Electrochem. Commun., 2, 235 (2000).

24. C. Amatore, F. Bonhomme, J. L. Bruneel, L. Servant, and L. Thouin, J. Electroanal. Chem., 484, 1 (2000).

25. T. Uematsu, J. T. Han, T. Tsuda, and S. Kuwabata, J. Phys. Chem. C, 116, 20902 (2012).

26. T. Kameyama, K. I. Okazaki, Y. Ichikawa, A. Kudo, S. Kuwabata, and T. Torimoto, Chem. Lett., 37, 700 (2008).

27. T. Torimoto, T. Adachi, K. Okazaki, M. Sakuraoka, T. Shibayama, B. Ohtani, A. Kudo, and S. Kuwabata, J. Am. Chem. Soc., 129, 12388 (2007).

28. K. Kano, Rev. Polarogr., 48, 29 (2002). 